\title{
Apperception: Understanding and Anticipating the User in Space Based on Neural and Behavioural Responses
}

\section{AUTHORS:}

Vladimír Šimkovič ${ }^{1}$

Ivan Kulifaj ${ }^{*}$

1, 2 Slovak University of Technology in Bratislava, Faculty of Architecture and Design, Institute of Public Buildings, Slovakia

\section{${ }^{*}$ CORRESPONDING AUTHOR}

E-mail: ivan.kulifaj@stuba.sk

\section{ARTICLE INFO}

Sent: Nov 23, 2020

Accepted: Mar 9, 2021

\begin{abstract}
:
The main task of architecture has been and will be to create spaces for the user. Our experiment is based on an interdisciplinary study, combining knowledge from architecture, neuroscience, psychology and artificial intelligence. We think that these disciplines can better interpret the users' perception to architects and designers, because how do we know what users want and how they react to the created matter? This fundamental question underlines the research, designates its direction and goals. The futuristic view and exploring the user is one aspect, complemented by the application of research results to today's reality. Nowadays, in Slovakia (and in the world), urban development is funded by the private sector and is significantly limited by the financial aspect of architecture and development, it is necessary to look for a concept that reduces the importance of finance at the expense of quality for users.
\end{abstract}

\section{KEYWORDS:}

neuroarchitecture, architecture, human perception, artificial intelligence, psychology

\section{PREFACE}

Our dissertation thesis presented in this paper examines a physical experiment, which is time-consuming, and organizationally and financially demanding. Unfortunately, due to COVID-19 and the closure of all institutions, our research has not been able to move beyond its theoretical stage, comprising methodology, procedures, organizational set-up and a broad search. In its current (as-is) stage, the experiment has partially covered the team formation (still ongoing), has completed technological search required for the laboratory work and negotiations with investors who are interested in the research. The experiment will move to the real-life phase at the moment when the pandemic be under control, as further requirements include personal meetings abroad.

\section{INTRODUCTION}

Seeking new perspectives, approaches and solutions has always been in the nature of humanity. Philosophical pondering over new concepts and futuristic considerations propels the evolution of humanity forward, and the pace accelerates. Certainly, there are different approaches applied with respect to the idea.

"If there really is no new way to be found, we are not afraid to stick with the old one that we found previously. So, I do not make every building different." (Ludwig Mies van der Rohe)

The main factor around which the topic of research is built is urban development. "Who participates in the city development process?" For the most part (in Bratislava it is 100\%), it is the private sector. Recently, the society has seen prevailing hostility against developers instead of looking for a way where both residents and the city on the one hand and the investor on the other would be satisfied. The topic of research and experiment also offer a solution to this problem, which also gives it the value of real use today. The ideal way where the tool to improve the quality of the environment and architecture, intended 
mainly for users or residents of the city, presents an interesting factor for the developer, bringing economic benefits, which are the primary attraction for "developers" of cities.

The answer to finding an appropriate balance that would suit both the developers, including architects, and the users, could be a new architectural branch, namely neuroarchitecture. Neuroarchitecture has an interdisciplinary nature, combining in itself the knowledge from neurosciences, which originally did not have any broader connection to traditional architecture or urbanism.

Neuroarchitecture is a discipline that examines how the physical environment that surrounds us can affect our brain on a neural basis, the way we feel when going through various experiences, and consequently our behaviour. This is a more of general explanation neuroarchitecture. As there is no need to enhance it as the final product of the research, architecture, or its approach is only based on neuroscientific data obtained from relationship between our brain and architecture.

The aim is to create a suitable combination of exterior and interior spaces, which would have a positive effect on the users' inner experience. It is the synergy of knowledge from disciplines such as architecture, neuroscience and psychology that can lead to the creation of spaces that will positively impact people's productivity and creative thinking, reduce stress and improve the quality of life of users who either live or work in or otherwise use such space.

This concept is a specific branch of architecture which aims at creating spaces that take into account the experience of their users. The very experience of users is largely based on their current perceptions. This area of scientific research is based on the fact that people spend more than $90 \%$ of time indoors [1]. In addition to the psychological experience itself, it is important to take into account the aspect of maximum utilization of space, because construction as such has an enormous impact on the environment. Hence, we believe that if we start to place more emphasis on what users feel and experience, we will achieve maximum use of non-renewable resources.

\section{DUAL ASPECT MONISM AS A THEORETICAL CON- CEPT IN ARCHITECTURAL RESEARCH}

When asked "How will the user behave?" it is important to find out how the human brain works in today's static architecture. Historically, mankind has been dealing with this issue since it abandoned a wandering lifestyle and the first intelligent civilizations appeared. There is a lot of diversification as to the space definition in order to achieve well-being. The topic has been part of philosophies of life, religions, reflected in geographical and cultural differences and, of course, in the numerous functional uses that have emerged in recent years and keep changing, for example, due to the global pandemic. Another, rational or scientific approach is to examine the individual through science, psychology, psychiatry, and neuroscience.

The theory of dual monism is a theoretical-philosophical concept, which we use to conceptualize our research. It could be said that the perception of the physical world can be measured in two ways: by questionnaire methods and observation, which can reveal the visible manifestations of the user, and neuroscientific methods, which capture the invisible manifestations of the user's experience. By combining these methods, we will obtain more comprehensive data on the experience and perception of users [2].

Dual monism is a philosophical concept that says the mental and physical worlds are two aspects of one reality. Unlike neutral monism, which claims that the mental as well as the physical dimension are two separate ways of organizing reality, which are neutral in themselves, that is, they are neither physical nor mental. This philosophical concept is only one of the many basic parts of the theoretical work prior to the experimental part.

\section{NEUROARCHITECTURE: AN INTERDISCIPLINARY APPROACH IN THE STUDY OF USER BEHAVIOR AND EXPERIENCE}

We believe that it is the merging of knowledge about human experience and behaviour from neuroscience with architecture that can help us better grasp the creation of spaces so as to maximize their utilization.

We can look at architecture from a psychological point of view and see it as a dimension of our consciousness that affects our experience. The connection between architecture and psychology can help us understand the invisible side of users in the form of their unconsciousness and the visible in the form of behaviour. If we include neuroscience in this concept, it can help us understand the user in terms of neurological changes in the brain. This model is illustrated in Fig. 1.

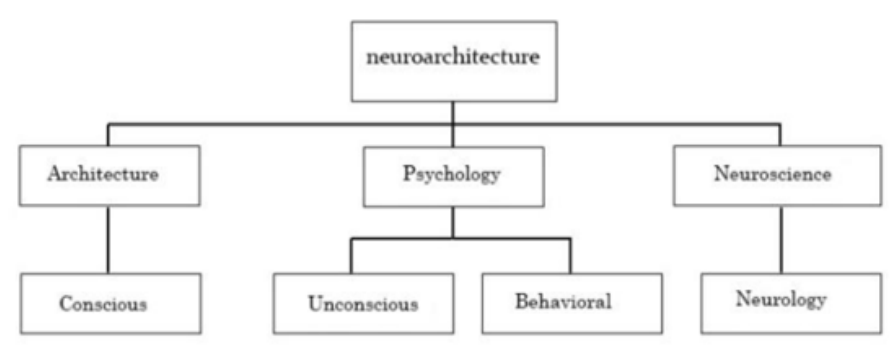

Figure 1: Neuroarchitecture as an interdisciplinary orientation and the disciplines of its subcategories [3]

According to Maleki and Bayzidi, applying an interdisciplinary approach can also impact the economic side of the project. According to the authors, the use of tools from the fields of neuroscience, such as $\mathrm{fMRI}$, which measures the metabolic activities of the brain; EEGs, which measure brain activity at the level of electronic neuron discharges or instruments that measure bodily manifestations such as the Eye Tracker, can help us capture user interactions with an object. Based on the findings of this equipment, we can determine the level of user interaction with the object. It is the understanding of this interaction that can help us to optimize the space so that it is used more.

Examples of an interdisciplinary approach used in the world include the construction of hospitals, offices or shopping centres. The home and urban environment is connected with our 
life experience and behaviour. These environments can acquire emotional and motivational value and subsequently shape our behaviour. Although there is well-known knowledge about the effects of space elements on perception, feelings, and affective responses [4] only limited attention has been paid to physical space-induced behaviour.

Similar topics are covered in several researches, which specialize either in one function (hospital) or in specific environments, such as virtual reality, interactive spaces or the gaming industry, or even artistic transformations of brain activity. The other works mentioned are primarily of a psychological nature dealing with the relationship between architecture and its impact on the human psyche.

"Step forward" or "Step towards" is a research conducted at the CMU School of Architecture, started in 2014. This research is both similar and different as to the specifics. Brief description: The work examines different views on the relationship between man and the environment, which are reflected in the responsive architecture. For the purposes of research, a particular system of responsive architecture was designed, analysed and subsequently applied in the experiment. The experiment consisted of subjects creating and responding to a digital version of matter or space that was projected and reacted back. Having analysed the interaction of people and artefacts it was concluded that a responsive architecture, regardless of its typology, builds its own worlds, which are valid only to their extent. The main specific of the experiment is that it did not address design or visuals at all [5].

A chapter in the book Culture and Gender based on the analytical psychology of Carl Jung, describes architecture as a structural diagram of the human psyche that counts and wins. The work analyses the importance and complexity of psychology in architecture, which in itself represents a multidisciplinary and complex craft. Finally, it concludes that by deepening the understanding of human experience of space, we can move the anthropocentric approach to design further; this being the initial concept of our thesis. Here, anthropocentric should not be understood in the sense of ignoring other aspects, but in the sense of improving quality [6].

Interactive Narratives for Responsive Architecture is an article from the Czech Republic, presenting the results of ongoing theoretical research into the phenomenon of interactive architecture. The main one is the exchange of information through social aspects, user experience and following the agent-based theory. Based on this, the publication states that interactive buildings require complex and consistent styles of interaction, not an isolated range of options (such as a sun-responsive façade, window shading...). It seeks to offer insights into the understanding of interaction systems [7].

The effect of colours on the human mind is one of the most explored phenomena, with generally believed truths such as "green has calming effects". Hence, green is often used in hospital interiors, waiting rooms or dispensaries. A study entitled The Effect of Color Psychology in Architecture examines the impact of colours and the environment based on the fact that colours have an immediate and long-term effect on human emotions. This study relies on book sources, analysis of technical documentation, and Internet articles on cyberspace. Its added value for the prepared experiment is that unlike a purely theoretical consideration it is rooted in reality. Certainly, this does not invalidate the research, which in fact serves very well as a partial basis for the experiment [8].

For us and the experiment, the most interesting research is the work of the artist Refik Anadol [9]. Using the word "research" is a very questionable concept in this case, as the author is an artist and the result is a work of art. Anadol pursues generative art and works with identical technology, however, in the exactly opposite way. Installations use brain activity when concentrated on certain phenomena, as a source of information; such as in the work "Melting Memories" it was the thoughts of an individual focused on memories. This activity was examined by a neurohelmet (as in the expected experiment) taking information from the brain and then transforming it into a 3D visual via an imaginary logarithm to create kinetic installations displayed by 3D holographic technology (again, similar to the experiment). Moreover, through their uniqueness and technological timelessness, the mentioned installations contain a script that translates information and produces its materialized form, thus imprinting in them the subjective view of the individual, who defines the whole process - that is the artist [9].

\section{THE ADVANTAGE OF AN INTERDISCIPLINARY AP- PROACH}

When creating a space, it is important to understand the conflicting views of different users of the space. For instance, this may relate to the construction of hospitals where potentially conflicting interests of users are at stake. Therefore, when building such a space, the cooperation of architect and psychologist can be beneficial, because we must take into account that on the one hand the space must act as a stimulus for doctors and other (medical) staff and at the same time consider the immediate interest of patients, which is rest and recovery.

By way of illustration, the main goal of hospitals is to offer adequate treatment for patients' recovery. For this reason, neurosciences are the basis of a balanced hospital architecture. The goal is to create an aesthetically pleasing environment and, at the same time, a functional environment aimed at improving the subjective experience of users' well-being. Given this, a neuroarchitectural approach can help meet the needs of different stakeholders and create a much more efficient building.

Furthermore, among other elements, the lighting had to be very intense in order to be able to quickly detect any changes in the newborn. Infants, on the other hand, had other needs. For example, it is the reception of appropriate stimuli so that the areas of the brain responsible for vision and hearing can develop properly. This was a very relevant point of conflict. Until the beginning of adulthood, brain development is marked by several "windows" associated with specific functions, such as balance, speech and hearing. The lack of adequate stimuli in these windows can permanently jeopardize the development of sensory and cognitive abilities. It was the neuro-architectural approach that helped reveal a potential conflict of interest for 
users. In new hospital facilities, the concept of neonatal intensive care units has changed radically to take into account the needs of children and the functional requirements of doctors and nurses [10].

The huge transit of people and their diversity remains a major challenge in hospital architecture. Likewise, the care and conditions that each group of patients requires are very specific and so the space given to them must fulfil different functions. We must take into account the interests and needs of healthcare professionals. During the operation, doctors must be able to concentrate and simultaneously communicate with their assistants. At the same time, in conversations about diagnoses and patient treatments, the environment must meet the conditions of privacy. Last but not least, the premises must take into account the possibility of rest for doctors and hospital staff.

\section{USER PSYCHOLOGY}

The basic neuropsychological functions of the brain include physiological arousal, attention, consciousness, decisionmaking processes, executive functions, language skills, learning, memory, motor coordination, perception, planning, problem solving, and thinking. It could be said that, apart from language skills, all other cognitive processes could be explored in connection with architectural space.

\section{A. Physiological arousal of the organism}

Arousal is a physiological and psychological state of awakening or sensory organs stimulated to the point of perception. It involves the activation of the ascending reticular activation system (ARAS) in the brain, which mediates wakefulness, the autonomic nervous system, and the endocrine system, leading to increased heart rate and blood pressure and state of sensory alertness, mobility, and readiness to respond.

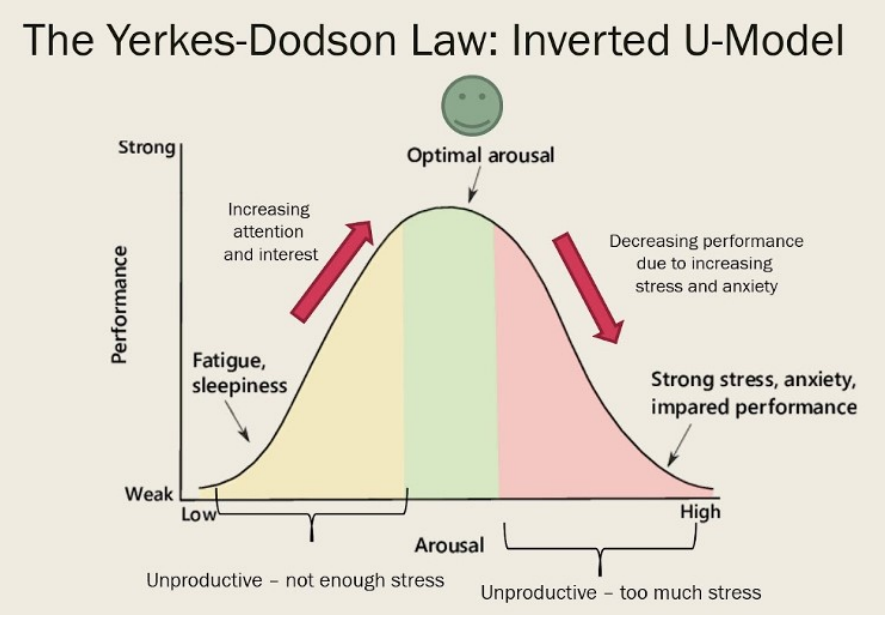

Figure 2: Yerkes-Dodson's law of optimal physiological arousal [11]

The arousal of the organism is important in the regulation of consciousness, attention, vigilance and information processing. It is essential to motivate certain types of behaviour, such as mobility, foraging, responding to a fight or flight, and sexual activity. Excitement is also important for the emotional response of the organism [12].
The degree of physiological arousal of the organism and its relationship with the virtual space was also examined in their study by Leite et al. through electrodermal activity and electrocardiogram [13]. The researchers did not find relevant differences in the degree of arousal between the tested groups. However, the study provides a good basis for the use of measuring the physiological arousal of the organism, the use of neuroscience tools and virtual reality [13].

\section{B. Attention}

Another important element that connects psychology, neuroscience and architecture is attention. Attention is a cognitive but also a behavioural process of selective concentration on stimuli. From the point of view of this work, however, we perceive the attention of users in terms of the kind of stimuli and how many stimuli the user is able to address [14].

\section{Consciousness}

The simplest way of consciousness could be defined as the perception of external and inner existence at a given moment. The issue of consciousness dealt with by psychology, medicine, philosophy, quantum physics, art and remains an unresolved query, whether in the material (interaction of consciousness with matter) or in the non-material (psychological, philosophical, etc.) world.

\section{Decision-making process}

In psychology, decision-making is considered a cognitive process leading to a choice from several possible alternatives, it can be either rational or irrational. The decision-making process is a reasoning process based on assumptions of the value system, preferences, and other factors that can influence a user's decision-making process (Simon, 2007). Each decisionmaking process leads to a final choice, which may or may not require an immediate action [15]. In the field of design and architecture, we see a great connection with the cognitive sciences and the work of Daniel Kahneman on fast and slow decision-making processes that can influence user behaviour.

\section{E. Perception}

Perception is the organization, identification and interpretation of sensory information in order to represent and understand the information presented or the environment [16]. The whole perception includes signals passing through the nervous system, which in turn are the result of physical or chemical stimulation of the sensory system.

Perception is not only the passive reception of signals, but is also shaped by the learning, memory, expectations and attention of the recipient. Sensory input is a process that transforms this low-level information into higher-level information (e.g. extracting shapes for object recognition) [17]. It is followed by the process of combining human concepts and expectations (or knowledge), regenerative and selective mechanisms (such as attention) that affect perception. 


\section{F. Memory}

Memory is the ability of the brain to encode, store, and retrieve data or information when needed. It is about storing information over time to influence future actions. If we could not remember past events, it would not be possible for us to develop language, relationships or personal identity.

Memory is often understood as a processing information system with explicit and implicit operation, which consists of a sensor processor, short-term (or working) memory and longterm memory. The sensor processor allows you to capture information from the outside world in the form of chemical and physical stimuli and address them at different levels of focus and intent. The working memory serves as an encoding and search processor. The stimulus information is encoded by the working memory processor in accordance with explicit or implicit functions. The working memory also reads information from previously stored material. Finally, the function of long-term memory is to store data using various categorical models or systems [18]. According to architect Alvar Alvarez, it is important to work with users' memory capacity when designing spaces [19].

The hippocampus is responsible for spatial memory. Every time we are in a space, primarily in one that we do not know, our brain processes the information into a spatial mental map that is hidden deep in our memory. We often do not look at the space in this way, but when we visit the same space again, we are somehow able to orient ourselves in it. Alvarez recommends creating spaces so that they are easy to remember for the user and outlines 5 basic physical stimuli that can help the user to better orientate himself in a space: depth, complexity, significant architectural elements, symmetry and repetitiveness [19].

\section{G. Emotionality}

In addition to the mentioned cognitive processes, it is important to focus on the emotional reaction of users with the stimulus (a product, or space in our case) within their user experience. Emotions are biological conditions associated with the nervous system, caused by neurophysiological changes that are variously associated with thoughts, feelings, behavioural reactions, and a degree of pleasure or dissatisfaction. There is currently no scientific consensus on the definition. Emotions often overlap with mood, temperament, personality, disposition, creativity and motivation [20].

Emotionality as part of the user experience was also addressed by Aaron Walter, author of Designing for Emotion, who used Maslow's hierarchy of needs to create a pyramid of users' needs. At the bottom of this pyramid, we can see the basic characteristics of any product-functionality (does this product work?). Then comes its reliability (is this product reliable?), usability (is this product easy to use?), and finally pleasurability (what makes us feel good when using this product?). "Pleasurable" products connect with users on an emotional level and this feature makes the users want to use the products even more [21].

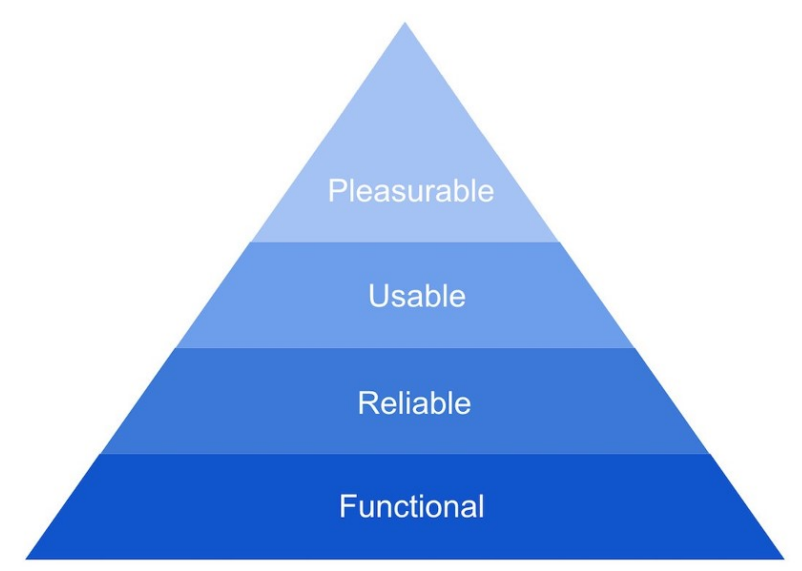

Figure 3: Pyramid of user needs according to Aaron Walter functionality—reliability—usability—pleasurability. [21]

We realize that the definitions described above do not embrace comprehensive data on cognitive processes. Nevertheless, they provide at least a basis for understanding the cognitive processes that affect the user's experience.

In the following chapter we describe the basic neuroscientific methods through which we can monitor and analyse user behaviour, such as Eye Tracker, which uses cameras to record the user's attention to individual stimuli, skin sensors to monitor emotional response and arousal or encephalography used to measure electrical activity.

\section{BASIC NEUROSCIENTIFIC METHODS THAT CAN BE USED IN ARCHITECTURE RESEARCH}

There are numerous neurological methods and we have chosen several basic ones, which have been repeated the most in various design or architectural studies. Alternatively, we are of the opinion that they could also be used in neuroarchitectonic research, in terms of their availability and prevalence.

\section{A. Functional magnetic resonance imaging (fMRI)}
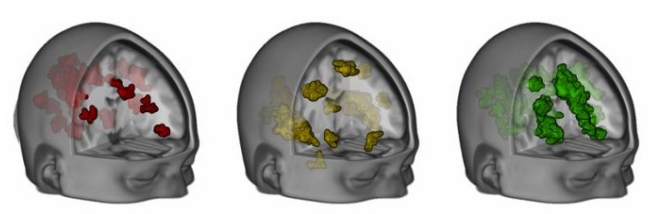

- content

- amusement

- surprise

- fear

- anger

sad

- neutral
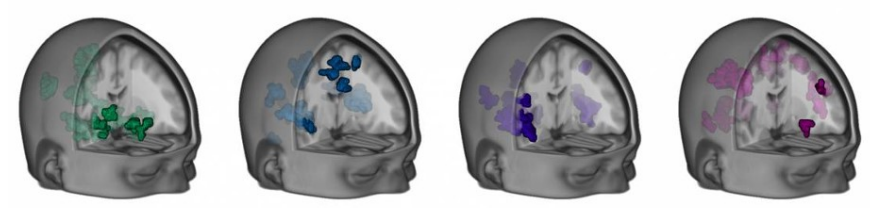

Figure 4: fMRI brain scan for emotional reactions of content, amusement, surprise, fear, anger, sadness, and neutral emotional reactions. [23]

Magnetic resonance imaging is currently the most advanced scanning tool for brain activity. This technique shows which part of the brain contains the most oxygenated blood, because if our brain performs an activity, it is the oxygenated blood that acts as fuel for it to perform that action. The blood carries oxygen and glucose to that part of the brain and so we can see on the display monitor which part of the brain is currently active [22]. 


\section{B. Electroencephalography (EEG)}

Electroencephalography is a method of creating an encephalogram record of the synaptic potentials of brain nerve cells. The EEG has several forms. The most commonly used version is the classic encephalograph (electroencephalograph in the narrower sense, scalp electroencephalograph), which is noninvasive and measures potentials on the surface of the scalp or stereoencephalogram, which means depth electrodes are inserted into parts of the brain. This method is used in surgery, while in research in the field of design and architecture, the classic version of the EEG is used [24]. EEG is probably the most widely used method in architectural or design studies which may be attributed to its affordability and ease of use. The use of EEG in architectural research is also documented in the quoted study [25].

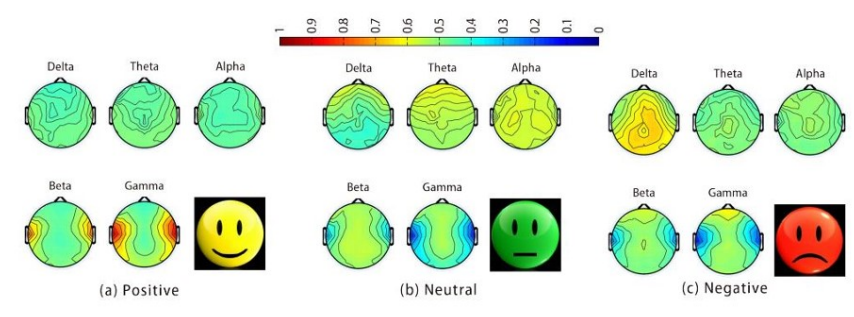

Figure 5: EEG brain scan of positive/neutral/negative emotional reactivity (valence) [26]

\section{Electrodermal activity (EDA)}

EDA, i.e. electrodermal activity is a property of the human body that causes constant changes in the electrical properties of the skin. EDA is a commonly used method in UX application and video game design (for example, in a study by Drachen and Nacke, 2010); other studies were developed in the field of architecture [27]. EDA and EKG were also used by Leite et al. [13], for measuring the physiological arousal of an organism in the form of anxiety, in response to a sense of security/danger from a virtual environment [28].

\section{Electromyography (EMG)}

EMG is an electrodiagnostic medical technique for evaluating and recording electrical activity produced by skeletal muscle(s).

Researchers Laparra-Hernández, Belda-Lois, Medina, Compos and Poveda [29] also set a limitation of the questionnaire measurement to obtain data from users. According to them, most product evaluations are based on data questionnaires when converting subjective data or user views. However, some users may feel slow or unable to make decisions when the differences in products are very subtle. Other techniques can contribute to understanding, such as physiological measurements of perception by including measurements that do not involve conscious processes.

In their research, they measured how users perceive different types of floor coverings in spaces. They used EMG and EDA for measurement. The results showed that the types of ceramic tiles could be distinguished based on EDA values, which showed differences in the emotional response of users. On the other hand, significant differences were found in EMG signals, especially as regards the facial muscle of the zygomaticus major. The results suggest that the analysis of this type of signal has potential for the use in understanding the perception of products by users.

\section{E. Electrocardiography (EKG)}

EKG is another non-invasive examination method that records the potentials generated by the heart muscle. Potentials are recorded by a device called an electrocardiograph (ECG) [30].

\section{F. Eye tracking}

Eye tracking is the process of measuring either the point of focus (i.e. where a person is looking) or the movement of the eye relative to the head. Eye Tracker is a device for measuring eye positions and eye movement. Eye trackers are used in UX design research of websites or applications. We believe that the ease of use and product availability can also be useful for design-architectural research [31].

We perceive that the use of the above-mentioned methods can help reveal differences in the subjective perception of products or spaces by their users and supplement the data from classical psychological questionnaire methods. Revealing "invisible" neuronal activity can help reveal differences in users' perceptions where they are unable to make decisions or perceive products very similarly.

\section{COGNITIVE SCIENCE AS A KEY TO UNDER- STANDING USER EXPERIENCE}

We can also look at the user evaluation of the product from the point of view of the cognitive sciences, specifically from the point of view of the user's decision-making processes. Daniel Kahneman, a Nobel Laureate in Behavioural Economics, came up with the concept of a fast and slow decision-making process, which he refers to as System 1 and System 2 [32].

System 1 is referred to as fast, automatic, emotional and subconscious. The System 1 thinking is reactive and responsible for complex but instinctive decision-making, which can include, for example, the assessment of the distance between objects or emotional reaction. This way of thinking is often referred to as "lazy" thinking.

System 2 is slow, strenuous and conscious. Operations such as logic or calculations take place in it. The System 2 thinking is analytical and applied to complex decision-making processes, such as determining appropriate social behaviour or comparing products in terms of price and performance [32].

We can also apply Kahneman decision-making and thinking systems to the product or space design. The human brain, for instance, does not want to process new information or make new decisions when faced with a relatively new scenario (we can notice this when deciding whether to stay on a new website or when we are in new premises). Therefore, people most often decide based on a quick system of thinking. In a fast-thinking system, the brain relies on learned patterns or mental shortcuts 
(heuristics) that allow us a high degree of adaptability but can lead to huge decision-making errors. If we make decisions based on system 1, there is a small probability that a slow decision system will "jump in". Users are not aware of these shortcuts, yet they have a significant impact on their perception, evaluation, and also interactions with products or the environment [32].

Thus, the brain prefers to make quick decisions, which ultimately makes UX product and environment design difficult. Slow user decision-making can also be captured through neuroscience methods [32].

\section{SIMILAR ARCHITECTURAL STUDIES USING KNOWLEDGE FROM PSYCHOLOGY AND NEUROSCIENCE}

Perception of design is a complex multifaceted process in which our touch, eyes and mind work together. Understanding the specific cognitive processes and forms of knowledge used in creative ways is essential. Neuroscience provides valuable tools for the analysis of thinking and design processes that complement traditional designer and architectural creation.

\section{A. Aesthetics of spaces}

The fact that our brain responds to various stimuli from our environment is also proven by an interdisciplinary study that examined how our brain influences the subjective evaluation of space aesthetics and the intention to either avoid or approach certain types of spaces [33]. The study used 200 different photographs of the spaces, which were presented to the participants. At the same time, the study used a differentiated inhouse design, i.e. first, their subjective perception of beauty was measured, and participants were to assess whether they liked the space or not. Subsequently, the same set of images was projected to them, but then they had to evaluate whether they would enter the space or not. During both measurements, the researchers monitored changes in brain activity by magnetic resonance imaging (fMRI).
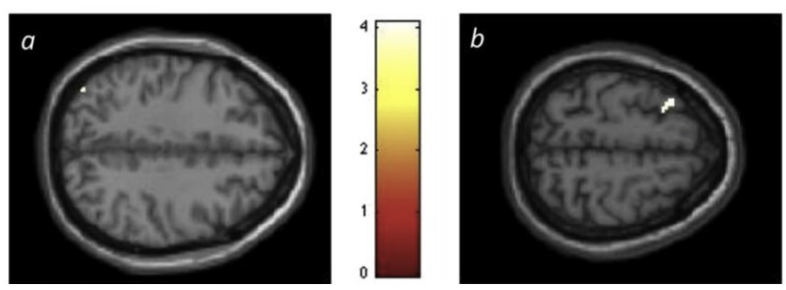

Figure 6: Changes in brain activity in probands' responses to high and low ceilings [33]

Seeing the rooms with a high ceiling, the part of the brain responsible for visual-spatial perception and attention, and also the dorsal area were activated in the participants. If they were shown rather open spaces, the fMRI showed as activated the brain area responsible for the visual perception of movement. When the participants were shown closed rooms, the anterior midcingulate cortex (aMCC) region, the cingulate gyrus region, and the amygdala region were activated.

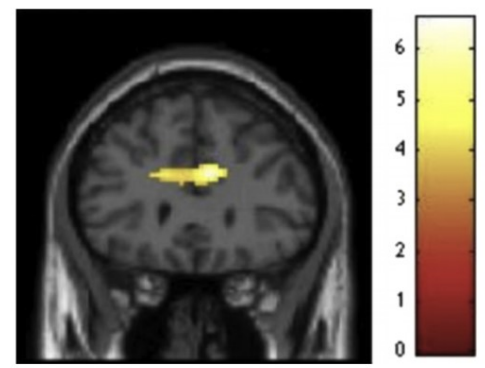

Figure 7: Activation of the cingulate cortex (aMCC), cingulate gyrus and amygdala in the presentation of enclosed spaces [33]

The results of the study show that in the subjective evaluation of the beauty of architectural objects, the parietal and frontal structures of the dorsal part of the brain are activated, which are responsible for visual-spatial perception and attention. This is relevant, though weak, evidence that the temporal lobes are also responsible for the subjective perception of beauty.

\section{B. Lighting temperature}

Using $\mathrm{fMRI}$ and psychological testing, respondents were asked to indicate the level of satisfaction on the Likert scale. In this way, Amor, O'Boyle and Pati wanted to learn about the effect of white colour temperature on the subjective perception of user satisfaction with space. They found that the warm white colour $(2,800 \mathrm{~K})$ did not show activation of the occipital cortex, which may indicate participants' disinterest in or dissatisfaction with the space. Nevertheless, the researchers noted the activation of certain parts of the brain with the cold spectrum $(4,100 \mathrm{~K})$; these were the upper frontal gyrus, which is also responsible for social interactions, the middle frontal gyrus, which is responsible for semantic and analytical operations, and the angular gyrus, responsible for recollection of memories and other cognitive functions. Even the cerebellum was activated during daylight, which, according to the latest neurological findings, is also largely responsible for cognitive processes and operations [34].

The use of knowledge from neurosciences in the field of design is currently very common, but there are only few studies on the use of neurosciences in the field of architecture; we could say that this trend is still emerging. According to us, architecture is an augmented form of product design and therefore we can draw plenty of key information from studies in this area.

\section{Spaces of the future}

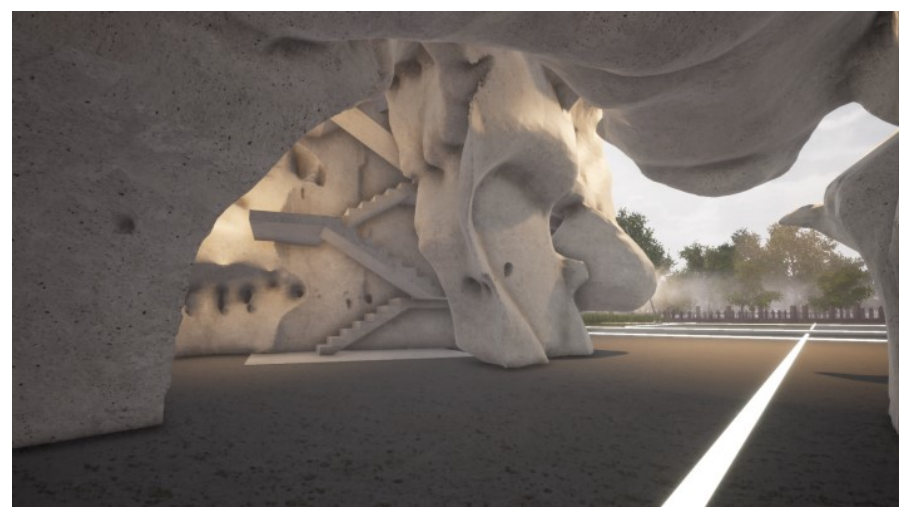

Figure 8: Space of the future [35] 
To understand more of the following a definition of space is needed. The Cambridge dictionary describes it as the "area around everything that exist, continuing in all directions". In our research philosophy, space is a 3-dimensional area around everything that can be observed by an individual, measurable by length scale or by time in unconscious and conscious way.

According to Frederick Marks, President of the Academy of Neuroscience for Architecture, "neurosciences are a rapidly evolving field whose primary focus is disease treatment and prevention, but has secondary implications for architecture and design." At the same time, Marks states that "The environment in which we live, work or play is constantly changing our brains and behavior. While the brain controls our behavior and genes control the plan of our brain, our environment can mediate the function of our genes and ultimately the structure of our brain." [36]

Amrita Kulkarni and Vincenzo Centinaro from the Gensler architecture firm also propose an interdisciplinary approach to space creation, claiming the creative behaviour in the form of critical thinking, problem solving, empathy, emotional intelligence and so on, are the most required skills in the modern workforce. They claim that when designing office space, we should take into account that employees should be able to develop this behaviour in the premises [37].

Marks sees the future in the interconnection of neurosciences and architecture or design. The growing knowledge from neuroscience disciplines only shows us why and how individual neuronal responses are triggered based on certain conditions. According to Marks, data on these processes can help us build a better and more interactive environment. Marks himself states that there is still a lack of studies on how our brains interact with the space or place where the user is located. According to Marks, designers and architects are the creators of the user experience that takes place through visual perception; haptic perception through, for example, skin response to different textures; sound perception and sound perception of space; what smells or scents evoke pleasure in us and at the same time the experience takes place through our memory abilities and the formation of associations. As stated by Marks, our understanding of these phenomena is another key to improving the design or architectural craft [36].

\section{EXPERIMENTAL PART}

We are aware of the complexity of our research, so we have so far described its theoretical model, which may slightly change based on the first testing. Our research is divided into two parts, the research part, consisting of data collection, and the final product, an artificial intelligence program for end users (architects or developers).

When devising the experimental design, we used a psychological research methodology that monitors the stimulus (specific stimuli), organismic (characteristics of the system processing the stimuli) and the response variables [37].
A) In our design, the stimulus variable is the digital environment presented through a VR headset. By digital environment we mean the presentation of existing or invented architectural spaces.

B) In our research, the organismic response is the user's response to stimuli, which will be measured by neurological methods.

C) The response variable means the users' responses recorded via a questionnaire.

When designing our research, we suggested 3 different alternatives of how the research could be conducted. In choosing neuroscientific methods, we used various studies that linked neurosciences with the user experience of space perception.

The goal of our research is to integrate data into the artificial intelligence program. We realized that setting up an interdisciplinary team for each project would be very time- and moneyconsuming, so our goal is to create a program using artificial intelligence technology, which would in a way replace psychological and neuroscientific testing for each project.

In our research, we decided to use the following neuroscientific methods:

EDA - measurement of skin conductivity to determine the physiological arousal of the body, which only determines the intensity (valence) of the experience; however, we are not able to determine whether the experience was positive or negative based on the data. The use of EDA is only relevant if it is combined with the EEG or EMG data.

EEG - activation of various brain centres (according to neurological maps we are able to identify which centres are behind which emotions, and also the valence of emotions); based on the range of brain waves we can determine the user's level of attention and interest in the stimulus (projection). The EEG has a high time resolution that allows it to match responses with corresponding stimuli.

ECG - measurement of heart rate changes, it works similarly to EDA. Again, ECG determines only the intensity of the organism's arousal, but the positivity/negativity of the experience cannot be determined from the data.

EMG - for unconscious facial movements, a principle similar to EDA.

Eye tracker - eye movement measurement (for example, determining which stimulus was the most visually attractive, etc.), Eye tracker is already built into modern VR headsets.

For data analysis, we will use iMotions software, which can analyse data from various tracking devices such as EEG, EDA, ECG and also EMG, and others. From our point of view, the optimal combination of methods is EDA + EEG + Eye Tracker + a simple questionnaire (Likert scale). 
In the following text, we describe 3 possible experimental designs, 2 of which do not involve the use of artificial intelligence.

\section{A. Design 1 without the use of artificial intelligence}

Design 1 makes it possible to measure all architectural parameters (lighting, colours, material, etc.) in different scales (intensity, source, etc.). The results measured can be used to extrapolate a standard, which could be supplemented by other parameters.

The design would only be used if intended as a long-term project and, at the same time it is not required to work with artificial intelligence. The disadvantages of design are that the result provided is not completely accurate; we can work only with a limited number of parameters that cannot be combined one with another and moreover, we are not able to obtain data from a series of consecutive situations.

\section{B. Design 2 - testing in real spaces}

This design model represents an extension of design 1 . In design 2, we do not examine individual parameters, but we test existing visualizations and how users feel in them. The created environment can be compared with others in order to find critical points of the design, which can be then modified and retested. This research design does not identify the causes, but can be helpful in finding the most suitable design alternatives. The disadvantage of this approach is that we can only work with current designs and knowledge transfer to other projects is not possible.

The disadvantage of both approaches is that they require a repeated formation of teams (a psychologist, a neuroscientist, and an architect) and repeated data collection. In order to avoid this aspect and optimize the working with data for other projects, we suggest using artificial intelligence, which will basically replace the work of neuroscientists and psychologists. We therefore propose to opt for the design 3, which, in its initial stages, is the most demanding as to the data, time, funds, and the research team.

\section{Design 3 using artificial intelligence to create a program for developers and architects}

The reason why we decided to choose design 3 for our research is its wide applicability. We also believe that it can help architects and developers to gain a better overview of user experience and adapt the product design accordingly. The result of the work of an interdisciplinary team should be the application of artificial intelligence, which will assign assumed user experience to various architectural parameters.

It is intended primarily for architects to test designs without the need to organize, for example, focus groups with different user sampling. The program will ensure that the maximum utilization of space is identified, i.e. it will combine the aesthetic view of architects with the experience and perception of users.

\section{CONCLUSION}

We are aware that it is not possible to include all theoretical psychological and neuroscientific starting points in the scope of the work, however, we are of the opinion the mentioned theories and research provide a solid basis for further research in the field of architecture and design. Furthermore, we also perceive the fact that the connection between neuroscience and psychology with design is still in its infancy, especially if we take into account studies originated in Slovakia and the Czech Republic. Therefore, drawing on psychology and neuroscience we tried to establish a basis, which could be used in the future and modified and updated later on as appropriate.

In this work, we have summarized several cognitive processes, which in our opinion are closely related to user experience, namely consciousness, memory, perception, decision-making, physiological arousal of the organism, attention, and emotionality. In further theoretical research, we would certainly focus on other cognitive processes, such as learning, the ability to plan and anticipate events, motor coordination, and problem solving. We believe these cognitive processes are also important in the user's interaction with space. Learning is largely related to our memory, including spatial memory, meaning that we can assume that user experience can also be influenced by how easy or difficult it is to remember a particular space. Alternatively, it can be affected by the way how the user may feel in similar premises (for example, the stores in the LIDL retail chain are arranged almost all the same). Planning and anticipating events is also an important cognitive factor, for example when designing spaces to serve users' quick actions, such as moving in the underground. Motor coordination can be a significant factor for people with various health (physical) disabilities, such as people using a wheelchair.

We realize that there are other psychological concepts, such as a typology of personality that could be measured in terms of user experience; still, we believe that cognitive processes and emotionality are more crucial in this concept. When conceptualizing research design, we stemmed from the assumption of an increased development/developers' activity and its impact on the environment, as well as the mental world of users. At the same time, we are working with the fact that neuroscience(s) and artificial intelligence are among the most developing fields of today.

The main goal is to deepen the understanding of the user's perception of space and thus push the method of design to a higher quality level. The basis of the research is a question arising from a futuristic concept, but the research findings are also applicable to contemporary static architecture, which is the main goal of our dissertation thesis. Today's application is mainly related to finding and offering the most ideal way to the construction market or developers. In this case criticism would be justified if the only objective were to help the private sector earn more, however, it is not true. The primary and major goal is to find new techniques and ways of designing architecture that serves people. The goal is to connect all segments that are working together in order to develop the city and enhance the entire process. 
The expected benefit of the work is to achieve a greater adaptation of today's architecture. This is one of the few researches that addresses human perception both through the conscious and the subconscious side of the subject, and both theoretically as well as through experiment (research). The work thus brings a different, more accurate view of the information about the user in space. This is further guaranteed by an interdisciplinary team of professionals who will bring views from other fields and crafts, from the perspectives of users, urban structure, and investors.

\section{REFERENCES:}

[1] Klepeis, N., Nelson, W., Ott, W., Robinson, J. (2001) "The National Human Activity Pattern Survey (NHAPS): A Resource for Assessing Exposure to Environmental Pollutants", Journal of Exposure Analysis and Environmental Epidemiology (Nature Publishing Group) (11), pp. 231-252.

https://doi.org/10.1038/sj.jea.7500165 [DOI]

[2] Atmanspacher, H., Fuchs, C. A. (2014) "The Pauli-Jung conjecture and its impact today“, Exeter: Imprint Academic.

[3] Maleki, M. R., Bayzidi, Q. (2018) "Application of Neuroscience on Architecture: The emergence of new trend of Neuroarchitecture", Kurdistan Journal of Applied Research, Vol. 3 (1) [online] Available at:

http://www.spu.edu.iq/kjar/index.php/kjar/article/view/131/131

10.24017/science.2018.1.62 [DOI]

[4] Baddeley, A., Eysenck, M. W., Anderson, M. C. (2015) "Memory", London, UK and New York, USA: Psychology Press. [5] Oh, Seoug (2014) "A step towards a Topology of Responsive Architecture: Actors, Interactions, Transformation", Electronic Theses and Dissertations for Graduate School. [online] Available at: https://etda.libraries.psu.edu/ [Accessed: 3 November 2020].

[6] Janetius, S.T. (2016) "Art, Culture and Gender: The Indian Psyche", Thrissur, Mishil and Js Publishers.

[7] Achten, H. (2019) "Interaction Narratives for Responsive Architecture", MDPI. [online] Available at:

https://www.mdpi.com/ [Accessed: 14 March 2019].

[8] Foladi, B. (2019) "The Effects of Color Psychology on Architecture", Academica.eu. [online] Available at:

https://www.academia.edu/ [Accessed: 2 November 2020].

[9] Anadol, R. (2019) "Machine Hallucination, Melting Memories, Infinite space", Refikanadol.com. [online] Available at: http://refikanadol.com/works/machine-hallucination/

[Accessed: 4 November 2020]

[10] Eberhard, J. (2009) "Brain Landscape: The Coexistence of Neuroscience and Architecture", Oxford: Oxford University Press, UK.

https://doi.org/10.1093/acprof:oso/9780195331721.001.0001 [DOI]

[11] Saeed, A., Trajanovski, S., Van Keulen, M., Erp, J. (2017) "Deep Physiological Arousal Detection in a Driving Simulator Using Wearable Sensors", IEEE ICDM workshops. New Orleans: Intl. Conference on Data Mining-workshop: Data Mining in Biomedical Informatics and Healthcare DMBIH.

[12] Mella, N., Conty, L., Pouthas V. (2011) "The role of physiological arousal in time perception: Psychophysiological evidence from an emotion regulation paradigm", Brain and Cognition 75 (2): pp. 182-187

https://doi.org/10.1016/j.bandc.2010.11.012 [DOI]

[13] Azevedo Leite, D. (2019) "Neo-Mechanistic Explanatory Integration for Cognitive Science: The Problem of Reduction Remains", ResearchGate. [online] Available at:

https://www.researchgate.net/ [Accessed: 2 November 2020].
[14] Anderson, J. R. (2005) "Cognitive psychology and its implications: John R. Anderson", Worth Publishers.

[15] Campitelli, G., Gobet, F. (2010) "Herbert Simon's Decision-Making Approach: Investigation of Cognitive Processes in Experts", Review of General Psychology 14 (4): pp. 354-364. https://doi.org/10.1037/a0021256 [DOI]

[16] Schacter, D., Gilbert, D., Wegner, D. (2011) "Sensation and perception", In: Psychology, by Charles Linsmeiser, Worth Publishers, pp. 158-159.

[17] Bernstein, D. (2010) "Essentials of Psychology", Cengage Learning.

[18] Baddeley, A., Eysenck, M. W., Anderson, M. C. (2015)

"Memory", Psychology Press, London, UK, and New York,

USA. https://doi.org/10.4324/9781315749860 [DOI]

[19] Alvarez, F. (2016.) "Francisco Alvarez Architecture Portfolio", ISSUU. [online] Available at: https://issuu.com/ [Accessed: 5 November 2020].

[20] Damasio, A. R. (1998) "Emotion in the perspective of an integrated nervous system", Brain Research Reviews 26 (2-3), pp. 83-86. https://doi.org/10.1016/S0165-0173(97)00064-7 [DOI]

[21] Walter, A. (2011) "Designing for Emotion", A Book Apart, New York, USA.

[22] Rinck, P. A. (2018) "Magnetic Resonance in Medicine", BoD.

[23] Kragel, P. A., LaBar, K. S. (2015) "Multivariate neural biomarkers of emotional states are categorically distinct", Social Cognitive and Affective Neuroscience 10 (11), pp. 1437-1448. https://doi.org/10.1093/scan/nsv032 [DOI]

[24] Hrazdira, I., Mornstein, V. (2001) "Lékařská biofyzika a přístrojová technika", Neptun. Czech Republic

[25] Banaei, M., Hatami, J. (2017) "Walking through Architectural Spaces: The Impact of Interior Forms on Human Brain Dynamics", Frontiers in Human Neuroscience, Vol. 477 (11). https://doi.org/10.3389/fnhum.2017.00477 [DOI]

[26] Zheng, W., Zhu, J., Lu, B. (2019) "Identifying Stable Patterns over Time for Emotion Recognition from EEG", EEE Transactions on Affective Computing 10 (3), pp. 417-429. https://doi.org/10.1109/TAFFC.2017.2712143 [DOI]

[27] Dias, M. S., Carreiro, M., Proença, P., Moural, A., Pedro, T., Freitas, J., Vilar, E., D'alpuim, J., Sérgio, A., Azevedo Sara Eloy (2014) "Designing Better Spaces for People. Rethinking Comprehensive Design: Speculative Counterculture, Proceedings of the 19th International Conference on Computer-Aided Architectural Design Research in Asia", Asia CAADRIA 2014. Hon Kong: The Association for Computer-Aided Architectural Design Research in Asia, pp. 739-748.

[28] Leite, S., Dias, M. S., Eloy, S., Freitas, J., Marques, S., Pedro, T., Ourique, L. (2019) "Physiological Arousal Quantifying Perception of Safe and Unsafe Virtual Environments by Older and Younger Adults", Sensors (Older and Younger Adults), Vol. 19 (11), pp. 2447. https://doi.org/10.3390/s19112447 [DOI] [29] Laparra-Hernández, J., Belda-Lois, J. M., Medina, E., Campos, N., Poveda, R. (2009) "EMG and GSR signals for evaluating user's perception of different types of ceramic flooring", International Journal of Industrial Ergonomics, Vol. 39 (2), pp. 326-332. https://doi.org/10.1016/j.ergon.2008.02.011 [DOI] [30] Lexico (2020) "EKG", Lexico. [online] Available at: https://www.lexico.com/en/definition/ekg [Accessed: 4 November 2020].

[31] Farnsworth, B. (2019) "What is Eye Tracking and How Does it Work?", Imotions. [online] Available at: https://imotions.com/blog/eye-tracking-work/ [Accessed: 2 November 2020].

[32] Kahneman, D. (2011) "Thinking, Fast and Slow", New York: Farrar, Straus and Giroux. 
[33] Vartanian, O., Navarrete, G., Chatterjee, A., Fich, L. B., Gonzalez-Mora, J. L., Leder, L., Modroño, C., Nadal, M., Rostrup, N., Skov, M. (2015) "Architectural design and the brain: Effects of ceiling height and perceived enclosure on beauty judgments and approach-avoidance decisions", Journal of Environmental Psychology 41, pp. 10-18.

\section{https://doi.org/10.1016/j.jenvp.2014.11.006 [DOI]}

[34] Amor, Cherif M., O'Boyle, M., Pati, D., Hou Jiancheng Duy Pham (2015) "Use of Neuroscience in Interior Design: Impact of Lighting Color Temperature on Attention Deficit Hyperactivity Disorder (ADHD) Subjects", Interior Design Educators Council. [online] Available at: https://www.idec.org/ [Accessed:

4 November 2020].

[35] Kulifaj, I. (2018) "Memoriál Železnej opony“, diploma work, Bratislava, STU, Slovakia.

[36] Schneider, A. (2020) "How the Application of Neuroscience in Design Can Separate Us from Artificial Intelligence", Interior Design. [online] Available at:

https://www.interiordesign.net/. [Accessed:

3 November 2020].

[37] Ferjenčík, J. (2010) "Úvod do metodologie

psychologického výzkumu“, Portál, Prague, Czech Republic. 